\title{
Black hole mass and binary model for BL Lac object OJ 287
}

\author{
F. K. Liu ${ }^{1,2}$ and X.-B. Wu ${ }^{2}$ \\ 1 Department of Astronomy and Astrophysics, Gothenburg University \& Chalmers University of Technology, \\ 41296 Gothenburg, Sweden \\ 2 Department of Astronomy, Peking University, 100871 Beijing, China \\ e-mail: wuxb@bac.pku.edu.cn
}

Received 7 February 2002 / Accepted 12 April 2002

\begin{abstract}
Recent intensive observations of the BL Lac object OJ 287 raise a lot of questions on the models of binary black holes, processing jets, rotating helical jets and thermal instability of slim accretion disks. After carefully analyzing their radio flux and polarization data, Valtaoja et al. (2000) propose a new binary model. Based on the black hole mass of $4 \times 10^{8} M_{\odot}$ estimated with the tight correlations of the black hole masses and the bulge luminosity or central velocity dispersion of host galaxies, we computed the physical parameters of the new binary scenario. The impact of the secondary on the accretion disk around the primary black hole causes strong shocks propagating inwards and outwards, whose arrival at the jet roots is identified with the rapid increase of optical polarization and the large change of polarization angle at about $0.30 \mathrm{yr}$ after the first main optical flare. An increase of optical polarization, a large rotation of positional angle and a small synchrotron flare at 2007.05 between the optical outbursts at 2006.75 and 2007.89 are expected by the model. With the estimated parameters, we predicated an increase of $\gamma$-ray flux appearing about 5 days after the first optical/IR peak, which is consistent with the EGRET observations.
\end{abstract}

Key words. galaxies: active - galaxies: BL Lac objects: individual: OJ 287 - galaxies: quasars: general accretion, accretion disk - black hole physics

\section{Introduction}

OJ $287(0851+202)$ at red-shift $z=0.306$ is one of the most active and best studied BL Lac objects. While it was identified in 1967 (Dickel et al. 1967), its optical observations were dated back to 1890s, which makes it be one of the best candidates for searching for long-term periodicity. Based on the historical optical light curves, Sillanpää et al. (1988) found the large optical outbursts of OJ 287 recurrent with a period of $11.65 \mathrm{yr}$ and proposed a binary black hole model, which predicted an outburst in the 1994 fall.

Although the predicted large optical outbursts were detected in 1994 and 1995, it was found that the outbursts are double-peaked and the binary black hole model has to be modified (Sillanpää et al. 1996). Among the proposed models to explain the periodicity of OJ 287, those of binary black holes (Lehto \& Valtonen 1996), procession jet (Katz 1997), rotating helical jets (Villata et al. 1998) and the thermal instability of slim accretion disk (Liu et al. 1995) can explain the periodicity in optical

Send offprint requests to: F. K. Liu, e-mail: fkliu@bac.pku.edu.cn band well. However, radio and polarization observations show that the first of the two peaks is narrow and thermal, and the second one is broad and synchrotronic (Valtaoja et al. 2000; Pursimo et al. 2000). Both flares should be synchrotronic and identical in the processing/helical jet models while thermal and similar to each other in the binary black hole model (Valtaoja et al. 2000). Another difficulty with the binary model is that it requires an extremely massive primary of $M=1.5 \times 10^{10} M_{\odot}($ Pietilä 1999), which is at least one order of magnitude larger than the super-massive black hole masses of other BL Lac objects (Barth et al. 2002; Wu et al. 2002; Falomo et al. 2002).

In the binary black hole model, the outburst thermal optical/IR photons should be up-scattered into $\gamma$-ray regime by the relativistic electrons in the jets via inverse Compton scattering process. From the parameters given by Pietilä (1999), the estimated time for photons to travel from the impact site to the jets is about 20 days and implies a delay of about three weeks between the optical/IR outbursts and high energy $\gamma$-ray radiations. Such a large lag is not consistent with the EGRET $\gamma$-ray observations (Shrader et al. 1996). 
To resolve the difficulties of the models mentioned above, Valtaoja et al. (2000) suggest a new binary scenario to explain the observations of radio flux and polarization. In their model, the first thermal flare of the outbursts is caused when a secondary black hole hyper-sonically passes through the accretion disk around the primary, and the second synchrotron flare after a viscous time scale $t_{\mathrm{vis}}$ is from the jet as the perigee passage enhances the accretion rate in the accretion disk, leading to increased jet mass flux and formation of strong shocks down the jet. As for such a scenario a constant period agrees with all the sharp outbursts (the first peak), a precession of the binary orbit and an extreme primary black hole are not needed (Valtaoja et al. 2000). However, as the lack of accurate knowledge of the primary black hole mass, Valtaoja et al. (2000) did not estimate the parameters of the new system. In this Letter, we extend Valtaoja et al's model to explain all the observations and try to estimate the physical parameters of the new scenario.

After a brief summary of the observations in Sect. 2, we describe the model in Sect. 3. In Sect. 4, we try to estimate the parameters of the model. Our discussions and conclusions are presented in Sect. $5 . H_{0}=50 \mathrm{~km} \mathrm{~s}^{-1} \mathrm{Mpc}^{-1}$ and $q_{0}=0.5$ are used throughout the paper.

\section{Observations}

The optical outbursts of OJ287 have a period of $P=$ $11.886 \mathrm{yr}$ (Kidger 2000) and are double-peaked with a spacing of 416 days (Sillanpää et al. 1996; Kidger 2000). The first flare is thermal and narrow without corresponding radio outburst and the second one is non-thermal and broad with strong outburst in radio (Valtaoja et al. 2000; Pursimo et al. 2000 and references therein). The variability in the IR band is similar to that in the optical band (Kidger et al. 1995; Pursimo et al. 2000). The first flare in the nearest 1994-1995 outbursts peaked at JD 1994.86 and the second one did at 1996.00. A small UV flare was detected during the first optical flare and no observation was made around the second one (Pursimo et al. 2000).

The observations made around JD 1994.88 showed no evidence for any X-ray outburst, but weak flux variations of time-scale as short as the time limit of the instrument (96 min) were detected (Idesawa et al. 1997; Pursimo et al. 2000). EGRET observations integrating from 10 to 15 November 1994 showed that in the $\gamma$-ray band the object was three times brighter than earlier, when it was in a low optical state (Shrader et al. 1996; Pursimo et al. 2000).

The optical polarization is very low during the first flare but very high during the second flare. A substantial increase of optical polarization and a large change of positional angle within one day at JD 1995.16 have been observed (Smith et al. 1987; Takalo 1994; Pursimo et al. 2000; Efimov et al. 2002). The variability of radio polarization is similar but with a time delay to that of optical polarization (Valtaoja et al. 2000; Pursimo et al. 2000).
The host galaxy of OJ 287 has been marginally resolved recently and has an absolute magnitude of $M_{\mathrm{R}}=$ -23.23 (Heidt et al. 1999)

\section{A revised binary black hole model}

The new binary scenario initially proposed by Valtaoja et al. (2000) and described in the introduction can be extended to explain the optical polarization, the X-ray variation and the $\gamma$-ray radiation of OJ 287.

In addition to an enhancement of the accretion rate, the perigee passage also causes strong shocks in the accretion disk, which propagate inwards and outwards at the speed of sound. The shocks arrive at the jet roots after a sound traveling time scale $\Delta t_{\mathrm{s}}$ and cause cause a very small increase of the jet flow and shocks down the jets, compressing the magnetic fields in the jets and therefore causing a rapid increase of the optical polarization and large rotation of positional angle about $\Delta t_{\mathrm{s}} \simeq 0.30 \mathrm{yr}$ after the first optical flare.

The thermal optical/IR photons of the first main flare feed the jets of the primary black hole and are up-scattered into the $\gamma$-ray regime by the relativistic electrons in the jets via inverse Compton scattering. The $\gamma$-ray outbursts, which have a delay of light traveling time from the impact site to the jets, may have been detected by EGRET. The detected weak X-ray variability may be generated around the secondary black hole, as the secondary is also an X-ray radiation source.

The apogee passage of the secondary black hole through the accretion disk may be too weak and undetectable.

\section{Parameters of the model}

\subsection{Masses of the black holes}

It was found recently that the masses of the center black holes in active and inactive galaxies follow tight correlations with the bulge velocity dispersions (e.g. Gebhardt et al. 2000; Merritt \& Ferrarese 2001a, 2001b) and the bulge luminosities (e.g. Magorrian et al. 1998; McLure \& Dunlop 2002). The host galaxy of OJ 287 was marginally resolved only recently of an effective radius $r_{\mathrm{e}}=0.72^{\prime \prime}$ and an absolute magnitude $M_{\mathrm{R}}=-23.23$ (Heidt et al. 1999). A black hole mass of $M \simeq 4.6 \times 10^{8} M_{\odot}$ is obtained with the absolute magnitude together with the correlation of black hole mass and bulge luminosity $\left(M-L_{\text {bulge }}\right)$ for active galaxies given by McLure \& Dunlop (2002). With the morphological data of the host galaxy in Heidt et al. (1999), we estimated the central velocity dispersion $\sigma$, using the fundamental plane of a tight correlation of velocity dispersion, effective radius and average surface brightness for elliptical galaxies (Bettoni et al. 2001). A second value of the black hole mass $M \simeq 3.2 \times 10^{8} M_{\odot}$ was derived with the estimated velocity dispersion $\sigma$ and the $M-\sigma$ correlation given by Merritt \& Ferrarese (2001b). The two estimated values of the black hole mass agree well with each 
other and are consistent with the upper limit $M \lesssim 10^{9} M_{\odot}$ obtained by Valtaoja et al. (2000). We used their mean value $M=4 \times 10^{8} M_{\odot}$ in the following calculation.

For the secondary, one can set only an upper limit $m / M_{\odot} \lesssim 10^{8}$, if the variability of the X-ray flux is from the accreting gas around it.

\subsection{Standard thin accretion disk or ADAF?}

While it has been suggested that the accretion disk in BL Lac objects is optically thin (Rees et al. 1982; Cavaliere \& D'Elia 2001), it is still possible that OJ 287 is exceptional and has an optically thick and geometrically thin accretion disk (e.g. Sundelius et al. 1997; Pietilä et al. 1999).

For an accretion disk, the viscous time-scale is $t_{\mathrm{vis}} \simeq$ $R / v \simeq R^{2} / \nu=R t_{\mathrm{s}} /(\alpha H)$ where $t_{\mathrm{s}}=R / c_{\mathrm{s}}$. If we take $t_{\mathrm{vis}} \simeq 1.14 /(1+z) \mathrm{yr}$ and $t_{\mathrm{s}} \simeq 0.30 /(1+z) \mathrm{yr}$, we have $H / R \gtrsim 0.3$ for $\alpha \lesssim 1$, which implies a geometrically thick disk. If we assume that large rotation of optical polarization angle (PA) around the time of the first optical outburst is caused by the passage of the secondary through the accretion disk, the nearly simultaneity between the PA rotation and optical outburst implies that the outburst radiation region becomes optically thin immediately when it is generated by the passage. So, it is naturally to assume the accretion disk being optically thin and geometrically thick, i.e. an advection-dominated accretion flow (ADAF).

For an ADAF, the self-similar solutions of sound speed $c_{\mathrm{S}}$ and radial velocity $v$ are (Narayan et al. 2000), respectively, $c_{\mathrm{s}}=c_{0} v_{\mathrm{K}}$ and $v=v_{0} v_{\mathrm{K}}$, where $v_{\mathrm{K}}$ is the Keplerian velocity, $c_{0}=(2 / 5)^{1 / 2}$ and $v_{0}=3 \alpha / 5$ for the politropic index $\gamma=5 / 3$. Therefore, we have

$\alpha=\frac{\sqrt{10}}{3} \frac{t_{\mathrm{s}}}{t_{\mathrm{vis}}}=0.28$

which is very close to the favored value $\alpha=0.25$ in literature (Narayan et al. 1998). The value of $\alpha$ slightly depends on the polytropic index $\gamma$ and we have $\alpha \simeq 0.31$ for $\gamma=7 / 5$.

\subsection{Parameters of the binary system}

If the secondary black hole is comparable to the primary, the primary would rotate around their mass center with a large amplitude, which leads up to in a significant sinusoidal twist of jets. Such a twist was not detected by VLBI (e.g. Tateyama et al. 1996; Roberts et al. 1987). So, we have $m \ll M$ and the major axis $a$ of the secondary orbit is

$a \simeq \frac{1}{2}\left(\frac{P c}{\pi r_{\mathrm{G}}(1+z)}\right)^{2 / 3} r_{\mathrm{G}}=4.1 \times 10^{2} M_{8}^{-2 / 3} r_{\mathrm{G}}$,

where $r_{\mathrm{G}}$ is the Schwarzschild radius of the primary and $M_{8}=M /\left(4 \times 10^{8} M_{\odot}\right)$.

The pericenter passage of the orbit is at radius

$R_{\min } \simeq c_{\mathrm{s}} t_{\mathrm{s}} /(1+z)=88 M_{8}^{-2 / 3} r_{\mathrm{G}}$.
If the accretion disk is a standard thin high- $\alpha$ disk, the orbit of the secondary may be coplanar with the accretion disk due to disk-secondary black hole interaction (Ivanov et al. 1998; cf. Valtaoja et al. 2000). However, the situation is unclear for an advection-dominated accretion flow, as the interaction between the secondary and the disk is negligible (Narayan 2000) and the coplanar time scale is of order of viscous time scale or larger (Ivanov et al. 1998; Scheuer \& Feiler 1996). In the following discussion, we assumed that the orbit of the secondary is roughly coplanar with the accretion disk and the secondary passes through the accretion disc twice per orbit. Then, the eccentricity of the orbit is $e \simeq 0.78$ and the apogee is at radius

$R_{\max }=7.2 \times 10^{2} M_{8}^{-2 / 3} r_{\mathrm{G}}$.

The IR/optical photons, which are up-scattered into $\gamma$-ray regime by the relativistic electrons in the jets, travel from the pericenter passage to the jets within a time scale $\Delta t_{\gamma} \simeq R_{\min } / c=4.0 M_{8}$ days. So, the model predicts an increase of $\gamma$-ray flux with a lag of $(1+z) \Delta t_{\gamma} \simeq 5$ days after the main flare, which is consistent with the EGRET observations (Shrader et al. 1996; Pursimo et al. 2000).

\subsection{The apogee passage}

From the self-similar solutions (Narayan et al. 2000), the ratio of the mass densities between the two passages is $\rho_{\max } / \rho_{\min } \simeq\left(R_{\min } / R_{\max }\right)^{3 / 2}=4.3 \times 10^{-2}$. As the radiation of optically thin hot gas is bremsstrahlung and proportional to the density square, the radiation is at least three order of magnitudes fainter at the apogee than at the pericenter. The difference of temperature, $T_{\max } / T_{\min } \sim$ $\left(R_{\min } / R_{\max }\right)=1.2 \times 10^{-1}$, makes the radiation at $R_{\max }$ even weaker and in lower frequency. Therefore, the burst at the apogee is undetectable.

\section{Discussions and conclusions}

Basing on the observations of OJ 287 in multi-frequencies and of the host galaxy, we extended and estimated the parameters of the revised binary model initially suggested by Valtaoja et al. (2000). In the model, the mass of the primary black hole is $4 \times 10^{8} M_{\odot}$, which is consistent with the upper limit obtained by Valtaoja et al. (2000) and about 40 times smaller than what the L\&V's model has given (Pietilä 1999). As the semi-major axis is, respectively, about $30 r_{\mathrm{G}}$ in the L\&V's model and about $4.1 \times 10^{2} r_{\mathrm{G}}$ in the revised model, the two black holes are about ten times closer in the L\&V's model than in ours (in units of the Schwarzschild radius). With such a less massive primary black hole and larger semi-major axis, the gravitational radiation is much weaker in the new scenario, though we cannot exactly estimate its value as we have only an upper limit of the companion mass. It can be expected that the lifetime of the binary system is much longer in the new model than that in the L\&V's which is only about $10^{4} \mathrm{yr}$. 
As we took the constant period of $P=11.886 \mathrm{yr}$ as in Kidger (2000), the predicted next outbursts (Kidger 2000) are that the first thermal optical flare is at 2006.75 (1 Oct. 2006) and the second synchrotron flare is at about 2007.89 (21 Nov. 2007). The predictions are consistent within the uncertainties of the period with those given by Valtaoja et al. (2000). In addition, an increase of optical polarization and large rotation of PA at 2007.05 (18 Jan. 2007) and brightening in $\gamma$-ray on 5 Oct. 2006 (at 2006.76) are expected by our model.

We identify the small X-ray variations observed about one week after the first flare with radiations from the hot gas around the secondary black hole. However, it is possible that the X-ray variability is not relevant to the pericenter passage event and cannot be detected during the 2006-2007 outbursts. Another possibility is that the arrival signal of sound wave traveling from the impact site to the jets is the variation in X-ray continuum instead of optical polarization. If so, an extremely high eccentricity $e=0.98$ is needed. The orbit of the secondary in such a system is completely embedded in the thick accretion disk and no outburst can be observed. Any binary system with such an extremely-high initial eccentricity would very rapidly reduce its eccentricity to $e \lesssim 0.8$ due to gravitational radiation and the interaction between the secondary and the accretion disk (cf. Artymowicz 1992).

If the traveling time scale of shock waves is as short as $t_{\mathrm{s}} / 2$, the eccentricity of the binary system is $e \simeq 0.87$ and most part of the orbit must be embedded in the accretion disk. No sharp optical outbursts can be detected. If the traveling time scale of the shock waves is longer than $t_{\mathrm{s}}$, e.g. $2 t_{\mathrm{s}}$, the orbit becomes very circular with $e=0.66$ and the two passages are very close to each other and $R_{\text {min }} / R_{\text {max }} \simeq 0.21$. The outbursts caused by the apogee passage become significant and detectable. In this case, an intersection of two periodic variabilities of $p=11.886 \mathrm{yr}$ should have been observed. One possibility is that the orbit is circular of $a \simeq 640 r_{\mathrm{G}}$ (note that the observed period $P_{\text {obs }}=P_{\text {orbit }} / 2$ ) and we have actually observed two equal passages with $R_{\min } \simeq R_{\max } \simeq a$. In this case, the traveling time scale of shock waves is about $7.3 t_{\mathrm{s}} \simeq 2.2 \mathrm{yr}$, which implies an unreasonably large $\alpha \simeq 2$.0. Our conclusion is that the shock traveling time scale of shock waves is between about $t_{\mathrm{s}} / 2$ and $2 t_{\mathrm{s}}$. It is reasonable to assume that the rapid increase of the optical polarization and the large rotation of positional angle after about $0.30 \mathrm{yr}$ following the first flare are caused by the shock waves arriving at the jet foot-points. As the shock waves may increase the mass flow into jet and cause shocks down the jets, a small synchrotron outburst corresponding to the change of optical polarization is also expected.

We have assumed that the orbit of the secondary is roughly coplanar with the accretion disk in the calculation of the eccentricity $e$ and $R_{\max }$. If they are not roughly coplanar with each other, the system would have a much higher eccentricity and closer apogee. This problem has already been discussed before.
We used the self-similar solutions to a stationary and vertically averaged one-dimension ADAF. Twodimensional numerical simulation (Igumenshchev \& Abramowicz 2000) showed that an ADAF with higher $\alpha$ $(\gtrsim 1)$ is convectively unstable. For an ADAF with lower viscosity $(\alpha \lesssim 0.1$ ), no bipolar or large scale circular motion is present and $t_{\text {vis }}$ should be passage-independent. For ADAF with a moderate viscosity $\alpha \sim 0.3$, while a self-similar solution is adequate, time-dependent bipolar outflows and large scale circulations are important (Igumenshchev \& Abramowicz 2000). Thus, a passagedependent time scale $t_{\mathrm{vis}}$ is not inconsistent with our model. However, to fully understand the time dependences of the inner accretion disk, two or even three dimensional numerical simulations are needed. In our model, a timedependent $t_{\mathrm{vis}}$ gives a time-dependent viscous parameter $\alpha$. Other estimated parameters are independent of $t_{\mathrm{vis}}$.

Acknowledgements. We are grateful to the anonymous referee for his/her helpful comments. FKL acknowledges support by the Swedish Natural Sciences Research Council (NFR). He thanks the director and staff of the Department of Astronomy and Astrophysics, Göteborg University \& Chalmers University of Technology, for their warm hospitality during his stay.

\section{References}

Artymowicz, P. 1992, PASP, 104, 769

Barth, A. J., Ho, L. C., \& Sargent, W. L. W. 2002, ApJ, 566, L13

Bettoni, D., Falomo, R., Fasano, G., et al. 2001, A\&A, 380, 471

Cavaliere, A., \& D'Elia, V. 2002, ApJ, in press [astro-ph/0106512]

Dickel, J. R., Yang, K. S., McVittie, G. C., \& Swenson, G. W. Jr. 1967, AJ, 72, 757

Efimov, Yu. S., Shakhovskoy, N. M., Takalo, L. O., \& Sillanpää, A. 2002, A\&A, 381, 408

Falomo, R., Kotilainen, J. K., \& Treves, A. 2002, ApJ, 569, L35

Gebhardt, K., Bender, R., Bower, G., et al. 2000, ApJ, 539, L13

Heidt, J., Nilsson, K., Appenzeller, I., et al. 1999, A\&A, 352, 11

Idesawa, E., Tashiro, M., et al. 1997, PASJ, 49, 631

Igumenshchev, I. V., \& Abramowicz, M. A. 2000, ApJS, 130, 463

Ivanov, P. B., Igumenshchev, I. V., \& Novikov, I. D. 1998, ApJ, 507,131

Katz, J. I. 1997, ApJ, 478, 527

Kidger, M. R. 2000, AJ, 119, 2053

Kidger, M. R., Gonzalez-Perez, J. N., de Diego, J. A., et al. 1995, A\&AS, 113, 431

Lehto, H. J., \& Valtonen, M. J. 1996, ApJ, 460, 207 (L\&V)

Liu, F. K., Xie, G. Z., \& Bai, J. M. 1995, A\&A, 295, 1

Magorrian, J., Tremaine, S., Richstone, D., et al. 1998, AJ, 115,2285

McLure, R. J., \& Dunlop, J. S. 2002, MNRAS, 331, 795

Merritt, D., \& Ferrarese, L. 2001a, MNRAS, 320, L30

Merritt, D., \& Ferrarese, L. 2001b, ApJ, 547, 140

Narayan, R. 2000, ApJ, 536, 663

Narayan, R., Igumenshchev, I. V., \& Abramowicz, M. A. 2000, ApJ, 539, 798 
Narayan, R., Mahadevan, R., Grindlay, J. E., Popham, R. G., \& Gammie, C. 1998, ApJ, 492, 554

Pietilä, H. 1998, ApJ, 508, 669

Pietilä, H., Takalo, L. O., Tosti, G., et al. 1999, A\&A, 345, 760

Pursimo, T., Takalo, L. O., Sillanpää, A., et al. 2000, A\&AS, 146,141

Rees, M. J., Phinney, E. S., Begelman, M. C., \& Blandford, R. D. 1982, Nature, 295, 17

Roberts, D. H., Gabuzda, D. C., \& Wardle, J. F. C. 1987, ApJ, 323,536

Scheuer, P. A. G., \& Feiler, R. 1996, MNRAS, 282, 291

Shrader, C. R., Hartman, R. C., \& Webb, J. R. 1996, A\&AS, 120,599

Sillanpää, A., Haarala, S., Valtonen, M. J., Sundelius, B., \& Byrd, G. G. 1988, ApJ, 325, 628

Sillanpää, A., Takalo, L. O., Pursimo, T., et al. 1996, A\&A, 315, L13
Smith, P. S., Balonek, T. J., Elston, R., \& Heckert, P. A. 1987, ApJS, 64, 459

Sundelius, B., Wahde, M., Lehto, H. J., \& Valtonen, M. J. 1997, ApJ, 484, 180

Takalo, L. O. 1994, Vistas Astron., 38, 77

Takalo, L. O. (ed.) 1996, Workshop on Two Years of Intensive Mointoring of OJ 287 and 3C 66A, Tuorla Obs. Rep. Informo 176 (Turku: Univ. Turku)

Tateyama, C. E., Inoue, M., Krichbaum, T. P., et al. 1996, PASJ, 48, 37

Valtaoja, E., Teräsranta, H., Tornikoski, M., et al. 2000, ApJ, 531,744

Villata, M., Raiteri, C. M., Sillanpää, A., \& Takalo, L. O. 1998, MNRAS, 293, L13

Wu, X.-B., Liu, F. K., \& Zhang, T. Z. 2002, A\&A, in press [astro-ph/0203158] 